\title{
Static Intervortex Forces
}

\author{
J.M. Speight \\ Department of Mathematics \\ University of Texas at Austin \\ Austin, Texas 78712, U.S.A.
}

\begin{abstract}
A point particle approximation to the classical dynamics of well separated vortices of the abelian Higgs model is developed. A static vortex is asymptotically identical to a solution of the linearized field theory (a Klein-Gordon/Proca theory) in the presence of a singular point source at the vortex centre. It is shown that this source is a composite scalar monopole and magnetic dipole, and the respective charges are determined numerically for various values of the coupling constant. The interaction potential of two well separated vortices is computed by calculating the interaction Lagrangian of two such point sources in the linear theory. The potential is used to model type II vortex scattering.
\end{abstract}

\section{Introduction}

The abelian Higgs model [1] is a relativistic field theory consisting of a complex scalar field $\phi$ coupled to a $U(1)$ gauge field $A_{\mu}$, and given a Higgs symmetry-breaking self interaction which allows topologically stable solitons to exist. The present work concerns the $(2+1)$-dimensional model, in which the solitons are simple lumps of energy called vortices (in $(3+1)$ dimensions the model admits extended string-like solitons [2] whose gravitational effects may be important in early Cosmology). Multivortex dynamics falls into one of three regimes, depending on the Higgs mass $\mu$ : if $\mu$ is small, vortices attract one another (the type I regime), while if $\mu$ is large they repel (type II), and at one critical value of $\mu$ static vortices exert no net force on one another.

In this paper, we calculate the interaction potential of two widely separated vortices by means of a novel approximation. The idea is that, viewed from afar, a static vortex looks like a solution of a linear field theory in the presence of a singular point source at the vortex centre. As will be shown, the appropriate point source is a composite scalar monopole and magnetic dipole in a KleinGordon/Proca theory. If physics is to be model independent, then the forces between well separated vortices should approach those between the corresponding point particles in the linear theory as the separation grows. Proceeding on this assumption, we calculate the asymptotic static two vortex potential. Our answer agrees with that of Bettencourt and Rivers, which was derived using a field superposition ansatz [3].

The monopole charge and dipole moment of the composite point source depend on the Higgs mass $\mu$. To fix their values, one must solve the static nonlinear field equations with vortex boundary conditions, and such solution is perforce numerical. Using a simple Runge-Kutta scheme we have determined these charges for various values of $\mu^{2}$. Here our results disagree with the work of Bettencourt and Rivers, who, while leaving undetermined the constants analogous to these charges, make certain assumptions about them which appear ill justified.

The asymptotic potential reproduces the aforementioned dynamical trichotomy into type I, type II and critical regimes found in the nonlinear model. As an application, the scattering of type II vortices is calculated and compared with numerical simulations. 


\section{The abelian Higgs model}

We begin by reviewing some standard results concerning the abelian Higgs model [1]. The Lagrangian density is

$$
\mathcal{L}=\frac{1}{2} D_{\mu} \phi \overline{D^{\mu} \phi}-\frac{1}{4} F_{\mu \nu} F^{\mu \nu}-\frac{\mu^{2}}{8}\left(|\phi|^{2}-1\right)^{2}
$$

where $D_{\mu} \phi=\left(\partial_{\mu}+i A_{\mu}\right) \phi$ is the gauge covariant derivative, $F_{\mu \nu}=\partial_{\mu} A_{\nu}-\partial_{\nu} A_{\mu}$ is the field strength tensor, and $\mathbb{R}^{2+1}$ has signature $(+,-,-)$. Note that the electric charge and vacuum magnitude of the Higgs field have been normalized to unity, leaving only one parameter $\mu$, the Higgs mass. With these conventions the model is critically coupled if $\mu=1$, and in the type I (type II) regime if $\mu<1$ $(\mu>1)$. The Euler-Lagrange equations derived from $\mathcal{L}$ are

$$
\begin{aligned}
D_{\mu} D^{\mu} \phi-\frac{1}{2} \phi\left(|\phi|^{2}-1\right) & =0 \\
\partial_{\mu} F^{\mu \nu}+\frac{i}{2}\left(\phi \partial^{\nu} \bar{\phi}-\bar{\phi} \partial^{\nu} \phi\right)+|\phi|^{2} A^{\nu} & =0
\end{aligned}
$$

a set of coupled, nonlinear, hyperbolic partial differential equations of which no nontrivial solutions are known.

If a configuration is to have finite energy, the fields should satisfy the following boundary conditions as $r=|\mathbf{x}| \rightarrow \infty$,

$$
|\phi| \rightarrow 1, \quad \mathbf{D} \phi \rightarrow \mathbf{0},
$$

whence $\phi_{\infty}:=\lim _{r \rightarrow \infty} \phi$ takes values on the unit circle in $\mathbb{C}$ and is thus a continuous map $S_{\infty}^{1} \rightarrow S^{1}$,

where $S_{\infty}^{1}$ represents the circle at spatial infinity. Such configurations fall into disjoint homotopy classes labelled by the degree of $\phi_{\infty}$ (an integer, $n$, also called the winding number of $\phi$ ). The time evolution defined by the field equations conserves energy, and so cannot take a field in one homotopy class into a different class, since such an evolution, being continuous, would define a homotopy between distinct classes, a contradiction. Hence $n$ is a topologically conserved quantity.

Another consequence of the boundary conditions is that the total magnetic flux is topologically quantized, for

$$
-\int d^{2} \mathbf{x} F_{12}=2 n \pi
$$

follows from (3) and Stokes' theorem. A static solution with $n=1$ is a stable lump of energy called a vortex. This may be visualized as a single flux tube in $\mathbb{R}^{3+1}$ with translation symmetry along the $x^{3}$ axis. It has a total flux of $2 \pi$ penetrating the physical plane.

\section{$3 \quad$ Vortex asymptotics}

The first task in the point particle approximation is to find out what a static vortex looks like far from its core 3, 4. We place the vortex at the origin, and use plane polar coordinates. Substituting the ansatz

$$
\begin{aligned}
\phi & =\sigma(r) e^{i \theta} \\
\left(A_{0}, A_{r}, A_{\theta}\right) & =(0,0,-a(r))
\end{aligned}
$$

( $\sigma$ is real) the field equations (2) reduce to two coupled nonlinear ordinary differential equations,

$$
\begin{aligned}
\frac{d^{2} \sigma}{d r^{2}}+\frac{1}{r} \frac{d \sigma}{d r}-\frac{1}{r^{2}} \sigma(1-a)-\frac{1}{2} \mu^{2} \sigma\left(\sigma^{2}-1\right) & =0 \\
\frac{d^{2} a}{d r^{2}}-\frac{1}{r} \frac{d a}{d r}+(1-a) \sigma^{2} & =0,
\end{aligned}
$$


the equations for $A_{0}$ and $A_{r}$ being trivially satisfied. Regularity demands that $\sigma(0)=a(0)=0$ while the boundary conditions (3) become

$$
\lim _{r \rightarrow \infty} \sigma(r)=\lim _{r \rightarrow \infty} a(r)=1 .
$$

Note that the ansatz has unit winding by construction. No exact solutions of (6) with these boundary conditions are known, but numerical solutions suggest that both $\sigma$ are $a$ are monotonic functions of $r$, and that the ansatz produces an isolated lump like structure.

We are interested in the asymptotic forms of $\sigma$ and $a$, and for these explicit expressions do exist. Define the functions $\alpha$ and $\beta$ such that

$$
\sigma(r)=1+\alpha(r), \quad a(r)=1+\beta(r) .
$$

Then (7) implies that $\alpha$ and $\beta$ are small at large $r$, so we substitute (8) into (6) and linearize in $\alpha$ and $\beta$,

$$
\begin{aligned}
\mu^{2}\left(\frac{d^{2} \alpha}{d(\mu r)^{2}}+\frac{1}{\mu r} \frac{d \alpha}{d(\mu r)}-\alpha\right) & =0 \\
\frac{d^{2}}{d r^{2}}\left(\frac{\beta}{r}\right)+\frac{1}{r} \frac{d}{d r}\left(\frac{\beta}{r}\right)-\left(1+\frac{1}{r^{2}}\right) \frac{\beta}{r} & =0 .
\end{aligned}
$$

These are the modified Bessel's equations of zeroth order for $\alpha$ in $\mu r$ and first order for $\beta / r$ in $r$ respectively. Hence, at large $r$,

$$
\begin{aligned}
& \alpha \sim \frac{q}{2 \pi} K_{0}(\mu r) \\
& \beta \sim \frac{m}{2 \pi} r K_{1}(r),
\end{aligned}
$$

where $K_{n}$ is the $n$-th modified Bessel's function of the second kind $\left[5\right.$. Note that $K_{1} \equiv-K_{0}^{\prime}$.

Since we have linearized the field equations, the asymptotic solutions contain unknown scale constants $q$ and $m$ which can only be fixed by solving (6) numerically. Rather than solving the boundary value problem $\sigma(0)=a(0)=0, \sigma(\infty)=a(\infty)=1$, we solve the initial value problem $\sigma(0)=a(0)=0$ using $\sigma^{\prime}(0)$ and $a^{\prime}(0)$ as shooting parameters. In fact, this is a slight oversimplification: due to the singularities of equations (6) at the origin, we must shoot from $r=r_{0}$, some small positive number, rather than $r=0$. Substituting Taylor expansions for $\sigma$ and $a$ into (6) we find that, near the origin,

$$
\begin{aligned}
\sigma & =a_{1} r+\frac{1}{4} a_{1}\left(b_{2}+\frac{\mu^{2}}{4}\right) r^{3}+O\left(r^{5}\right) \\
a & =b_{2} r^{2}-\frac{a_{1}^{2}}{8} r^{4}+O\left(r^{5}\right) .
\end{aligned}
$$

We use $a_{1}$ and $b_{2}$ as shooting parameters, adjusting them until the numerical solution has $\sigma\left(r_{\infty}\right) \approx$ $1 \approx a\left(r_{\infty}\right)$, where $r_{\infty}$ is some large positive number, the effective infinity. Having generated such a numerical solution, we compare it at large $r$ to the asymptotic forms (10) and deduce $q$ and $m$.

The results of this procedure using a fourth order Runge-Kutta method with $r_{0}=10^{-8}$ and $r_{\infty}=10$ for various values of $\mu^{2}$ are presented in table 1 . That $r_{\infty}$ is so small is unfortunate but necessary: at large $r$ the field equations reduce to Bessel's equations, which have two independent solutions, one exponentially decaying and the other exponentially growing. We seek to pick out the former and completely exclude the latter, an impossible task. Hence, all numerical solutions blow up at large $r$, and even though $a_{1}$ and $b_{2}$ were tuned to six decimal places, the Runge-Kutta algorithm could not shoot beyond $r=10$. 


\begin{tabular}{|c|c|c|c|}
\hline$\mu^{2}$ & $q$ & $m$ & $r_{c}$ \\
\hline 0.4 & -7.54 & -14.92 & 4.23 \\
0.6 & -8.71 & -12.61 & 3.75 \\
0.8 & -9.70 & -11.31 & 3.43 \\
0.9 & -10.14 & -10.89 & 3.22 \\
1.0 & -10.58 & -10.57 & - \\
1.1 & -10.98 & -10.31 & 2.98 \\
1.2 & -11.43 & -10.06 & 3.07 \\
1.3 & -11.80 & -9.85 & 2.96 \\
1.4 & -12.23 & -9.66 & 2.95 \\
1.6 & -13.04 & -9.34 & 2.88 \\
1.8 & -13.97 & -9.09 & 2.87 \\
2.0 & -14.50 & -8.86 & 2.72 \\
\hline
\end{tabular}

Table 1: Numerical values of vortex scalar charge $q$ and magnetic dipole moment $m$. The other data are the critical points of the static intervortex potential.

Nevertheless, the qualitative nature of the $\mu^{2}$ dependence of $q$ and $m$ is clear. In particular, two points about the numerical charges are noteworthy. First, at critical coupling $\left(\mu^{2}=1\right), q \approx m$. In fact, one can prove that $q \equiv m$ exactly in this case, because the static $\mu^{2}=1$ vortex satisfies a pair of first order field equations. These are deduced by means of an argument due to Bogomol'nyi [6],

$$
\begin{aligned}
\left(D_{1}+i D_{2}\right) \phi & =0 \\
-F_{12}+\frac{1}{2}\left(|\phi|^{2}-1\right) & =0
\end{aligned}
$$

(in the $A_{0}=0$ gauge) and within our ansatz take the form [7],

$$
\begin{aligned}
r \frac{d \sigma}{d r}-(1-a) \sigma & =0 \\
\frac{2}{r} \frac{d a}{d r}+\left(\sigma^{2}-1\right) & =0 .
\end{aligned}
$$

Substituting (8) into (14) yields, on linearizing,

$$
\beta=-r \frac{d \alpha}{d r}
$$

and so $\alpha=q K_{0}(r) / 2 \pi \Rightarrow \beta=q r K_{1}(r) / 2 \pi$. Thus $m \equiv q$. We emphasize that this argument works only at critical coupling.

Second, $|q|$ and $|m|$ are monotonic functions of $\mu^{2},|q|$ increasing and $|m|$ decreasing. Bettencourt and Rivers [3] also find the asymptotic forms (10), but leave their charges analogous to $q$ and $m$ undetermined. For purposes of calculation, they make two assumptions about the charges which, in the light of table 1, may prove ill-justified. First, they assume that $q=m$ is approximately true away from $\mu^{2}=1$, whereas in our results, $q / m$ varies between 0.50 and 1.64 . Second, they impose the condition that the magnetic flux of a vortex should vanish at $r=0$ and deduce that $m=-2 \pi$ (it is unclear why a condition at $r=0$ should directly constrain the asymptotic behaviour as $r \rightarrow \infty)$. This result may be valid for very large $\mu^{2}$, but is certainly flawed close to $\mu^{2}=1$ since the $\mu^{2}=1$ vortex has maximum magnetic flux at the origin, as is easily seen from the lower Bogomol'nyi equation (13). So, they combine assumptions which are individually true only in widely disparate physical regimes. 


\section{The point vortex}

The next task is to replicate the vortex asymptotics, found above, in the linear field theory by coupling the fields in standard fashion to a scalar density $\rho$ and a vector current $j_{\mu}$, as yet undetermined. To linearize the abelian Higgs model, we choose gauge so that $\phi$ is real. Defining the field $\psi=1-\phi$, the vacuum is then $\psi=0$, and the linear Lagrangian density is obtained by expanding (1) up to quadratic order in $\psi$ and $A_{\mu}$,

$$
\mathcal{L}_{\text {free }}=\frac{1}{2} \partial_{\mu} \psi \partial^{\mu} \psi-\frac{1}{2} \mu^{2} \psi^{2}-\frac{1}{4} F_{\mu \nu} F^{\mu \nu}+\frac{1}{2} A_{\mu} A^{\mu} .
$$

Including the external source Lagrangian density,

$$
\mathcal{L}_{\text {source }}=\rho \psi-j_{\mu} A^{\mu},
$$

we obtain the following massive, inhomogeneous wave equations for $\psi$ and $A_{\mu}$,

$$
\begin{aligned}
& \left(\square+\mu^{2}\right) \psi=\rho \\
& (\square+1) A_{\mu}=j_{\mu}+\partial_{\mu}\left(\partial_{\nu} j^{\nu}\right) .
\end{aligned}
$$

All gauge freedom has been exhausted, and there is no global $U(1)$ symmetry of $\mathcal{L}_{\text {free }}$ with whose Noether current we can identify $j_{\mu}$ because $\psi$ is real. Hence there is no reason to assume that $j_{\mu}$ is a conserved current, and we cannot set the extra "fictitious current" term in the Proca equation (20) to zero.

To make comparison with the asymptotic vortex fields, these must first be converted to the real $\phi$ gauge. Since $\phi$ has non-zero winding, there is no gauge transformation regular on all $\mathbb{R}^{2}$ which will accomplish this. However, we only require comparison at large $r$, so for our purposes it is sufficient that the transformation be regular on $\mathbb{R}^{2} \backslash\{\mathbf{0}\}$. Since a singular point source will be introduced into the linear theory, this is from the outset regular only on $\mathbb{R}^{2} \backslash\{\mathbf{0}\}$. So, we unwind the static vortex (5) with gauge transformation $\phi \mapsto e^{-i \theta} \phi, A^{\mu} \mapsto A^{\mu}+\partial^{\mu} \theta$ to obtain

$$
\begin{aligned}
\phi & =\sigma(r) \sim 1+\frac{q}{2 \pi} K_{0}(\mu r) \\
A_{\theta} & =-a(r)+1 \sim \frac{m}{2 \pi} r K_{1}(r),
\end{aligned}
$$

while $A_{r}=A_{0}=0$. It is convenient to introduce a unit vector $\widehat{\mathbf{k}}$ in a fictitious third direction perpendicular to the physical plane, so that the $\mathbb{R}^{3}$ vector product can be defined. In terms of the 2 -vector field $\mathbf{A}$, the unwound asymptotic behaviour is

$$
\mathbf{A} \sim-\frac{m}{2 \pi} K_{0}^{\prime}(r) \widehat{\boldsymbol{\theta}}=-\frac{m}{2 \pi} \widehat{\mathbf{k}} \times \nabla K_{0}(r) .
$$

We thus seek sources $\rho$ and $j_{\mu}$ such that the solutions of (19,20) are

$$
\begin{aligned}
\psi & =\frac{q}{2 \pi} K_{0}(\mu r) \\
\left(A^{0}, \mathbf{A}\right) & =\left(0,-\frac{m}{2 \pi} \widehat{\mathbf{k}} \times \nabla K_{0}(r)\right) .
\end{aligned}
$$

The static Klein-Gordon equation in $(2+1)$ dimensions has Green's function $K_{0}$,

$$
\left(-\Delta+\mu^{2}\right) K_{0}(\mu r)=2 \pi \delta(\mathbf{x}) .
$$

Substituting (24) into (19) and using (26) one finds that

$$
\rho=q \delta(\mathbf{x}) .
$$


Similarly, substitution of (25) into (20) yields

$$
\mathbf{j}-\nabla(\nabla \cdot \mathbf{j})=-m \widehat{\mathbf{k}} \times \nabla \delta(\mathbf{x}) .
$$

Taking the divergence of (28) one sees that $\nabla \cdot \mathbf{j}$ is a solution of the homogeneous static Klein-Gordon equation, so if $\mathbf{j}$ is a point source (meaning $\mathbf{j}=\mathbf{0}$ except at $\mathbf{x}=\mathbf{0}$ ) then $\nabla \cdot \mathbf{j}=0$ everywhere. Thus the unique point source satisfying (28) is

$$
\mathbf{j}=-m \widehat{\mathbf{k}} \times \nabla \delta(\mathbf{x}) .
$$

Since $A^{0}=0$ we take $j^{0}=0$. The physical interpretation of these expressions for $\rho$ and $\mathbf{j}$ is that the point source consists of a scalar monopole of charge $q$ and a magnetic dipole of moment $m$ perpendicular to the physical plane. Both $q$ and $m$ are negative (see table 1). We refer to this composite point source as the point vortex.

\section{The static intervortex potential}

Having found the scalar charge and magnetic dipole moment carried by a point vortex, it is straightforward to calculate the force between two such vortices held at rest, in the framework of the linear theory. The interaction Lagrangian for two arbitrary (possibly time dependent) sources $\left(\rho_{1}, j_{(1)}\right)$ and $\left(\rho_{2}, j_{(2)}\right)$ is

$$
L_{\mathrm{int}}=L_{\psi}+L_{A}=\int d^{2} \mathbf{x} \rho_{1} \psi_{2}-\int d^{2} \mathbf{x} j_{(1)}^{\mu} A_{\mu}^{(2)}
$$

where $\left(\psi_{i}, A_{(i)}\right)$ are the fields induced by source $\left(\rho_{i}, j_{(i)}\right)$ according to the wave equations (19,20). This is found by extracting the cross terms in $\int d^{2} \mathbf{x}\left(\mathcal{L}_{\text {free }}+\mathcal{L}_{\text {source }}\right)$ where $(\rho, j)$ is the superposition of the two sources, and $(\psi, A)$ is a superposition of the induced fields. The expression (30) looks asymmetric under interchange of sources $1 \leftrightarrow 2$, but in fact $L_{\text {int }}$ is symmetric as may be shown using the wave equations $(19,20)$ and integration by parts.

Now consider the case of two static point vortices, vortex 1 at $\mathbf{y}$ and vortex 2 at $\mathbf{z}$. Then $\rho_{1}=q \delta(\mathbf{x}-\mathbf{y})$, while the scalar field due to $\rho_{2}$ is $\psi_{2}=q K_{0}(\mu|\mathbf{x}-\mathbf{z}|) / 2 \pi$. Hence,

$$
L_{\psi}=\int d^{2} \mathbf{x} \frac{q^{2}}{2 \pi} \delta(\mathbf{x}-\mathbf{y}) K_{0}(\mu|\mathbf{x}-\mathbf{z}|)=\frac{q^{2}}{2 \pi} K_{0}(\mu|\mathbf{y}-\mathbf{z}|) .
$$

The magnetic interaction is similar: $j_{(1)}^{0}=0, \mathbf{j}_{(1)}=-m \widehat{\mathbf{k}} \times \nabla \delta(\mathbf{x}-\mathbf{y})$ while $A_{(2)}^{0}=0, \mathbf{A}_{(2)}=$ $-m \widehat{\mathbf{k}} \times \nabla K_{0}(|\mathbf{x}-\mathbf{z}|)$, so

$$
\begin{aligned}
L_{A} & =\int d^{2} \mathbf{x} \frac{m^{2}}{2 \pi}[\widehat{\mathbf{k}} \times \nabla \delta(\mathbf{x}-\mathbf{y})] \cdot\left[\widehat{\mathbf{k}} \times \nabla K_{0}(|\mathbf{x}-\mathbf{z}|)\right] \\
& =-\frac{m^{2}}{2 \pi} \Delta_{y} K_{0}(|\mathbf{y}-\mathbf{z}|) \\
& =-\frac{m^{2}}{2 \pi} K_{0}(|\mathbf{y}-\mathbf{z}|)
\end{aligned}
$$

using (26) with $\mathbf{y} \neq \mathbf{z}$. The total interaction Lagrangian is a function of $|\mathbf{y}-\mathbf{z}|$ only, so we interpret $-L_{\text {int }}$ as the potential energy of the interaction,

$$
U=\frac{1}{2 \pi}\left[m^{2} K_{0}(r)-q^{2} K_{0}(\mu r)\right]
$$

where $r$ is the vortex separation, that is $\mathbf{r}=r(\cos \vartheta, \sin \vartheta):=\mathbf{y}-\mathbf{z}$. This is the same potential as found in [3], but we arrived at it via a different route. 
This potential is consistent with the partition into type I, critical and type II regimes. The central force due to $U$ is

$$
-U^{\prime}(r)=\frac{1}{2 \pi}\left[m^{2} K_{1}(r)-\mu q^{2} K_{1}(\mu r)\right] .
$$

If $\mu<1$, then $K_{1} \rightarrow 0$ at large $r$ faster than $K_{1}(\mu r)$, so scalar attraction dominates over magnetic repulsion and the force is negative, consistent with type I behaviour. If $\mu>1$, the reverse is true and the force is positive at large $r$, consistent with type II behaviour. Potentials for $\mu^{2}=0.4$ (type I) and $\mu^{2}=2.0$ (type II) are plotted in figure 1 . At $\mu=1, m \equiv q$, as explained in section 3 so $U \equiv 0$ and there is no net force at all. This consistency at large $r$ emerges regardless of the specific values of $m$ and $q$ away from $\mu=1$, and may be attributed to the inverse relationship between a field's mass and its range. At moderate $r$, the $\mu$ dependance of $q / m$ becomes important. Given that $K_{1}$ is a strictly decreasing function, it is clear from (34) that there exists a unique critical point of $U$ for each $\mu \neq 1$ if and only if $m / q>\sqrt{\mu}$ when $\mu<1$ and $m / q<\sqrt{\mu}$ when $\mu>1$. Our numerical work suggests that $m / q$ easily passes these criteria. The rightmost column of table 1 presents the approximate critical vortex separation $r_{c}$ for each value of $\mu^{2}$. No such critical points were found by Rebbi and Jacobs [8], who obtained approximate static intervortex potentials by numerically minimizing the potential energy functional subject to the constraint that $\phi$ has two simple zeros separated by a given distance. So these equilibria are probably artifacts of our approximation, which we take to break down for $r \leq r_{c}$. Of course, some kind of breakdown is to be expected: vortices are not point particles, as in our picture, and when they approach one another closely enough their overlap produces significant effects.

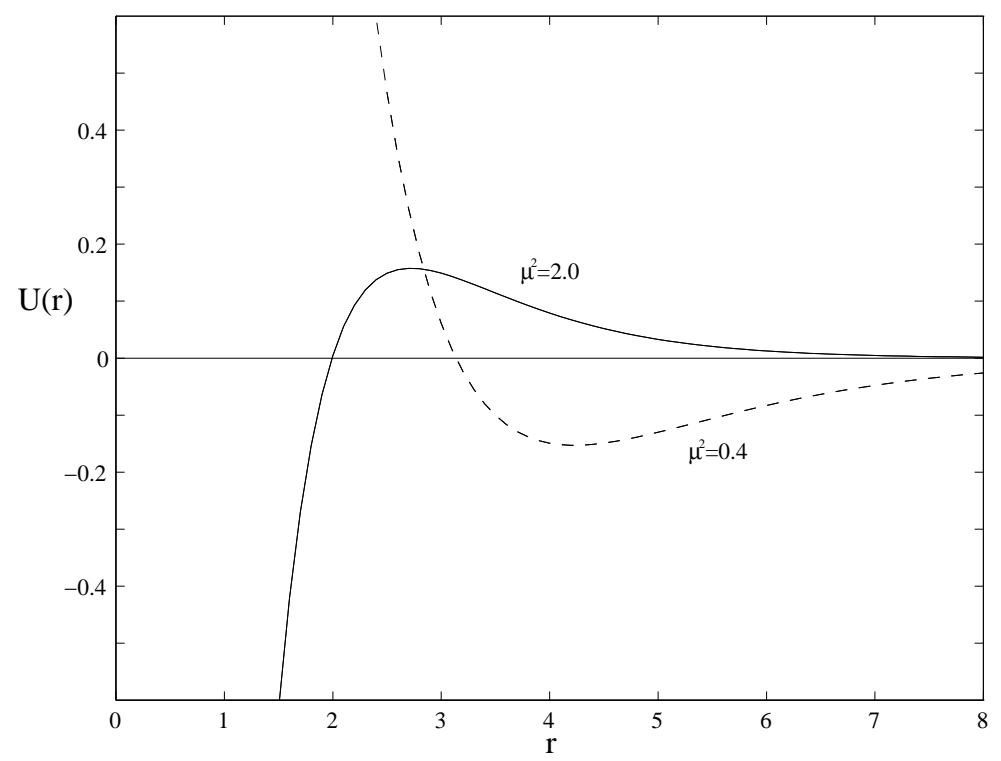

Figure 1: The potential function $\mathcal{U}(r)$ for $\mu^{2}=0.4$ and $\mu^{2}=2.0$.

\section{$6 \quad$ Type II vortex scattering}

The interaction potential $U$ provides a very simple model of two-vortex dynamics: the dynamics of two point particles each of mass $M$ (the energy of a single vortex at rest, a $\mu^{2}$ dependent quantity) interacting via the potential (33). Ignoring the (trivial) centre of mass motion, the Lagrangian of such a mechanical system is

$$
L=\frac{1}{4} M\left(\dot{r}^{2}+r^{2} \dot{\vartheta}^{2}\right)-U(r)
$$


since the reduced mass of the system is $M / 2$. This is a manifestly bad model if $\mu=1$, because it would predict that there is no scattering at all, in conflict with the results of numerical simulations [9] and the geodesic approximation [7]. Away from critical coupling, one might expect the potential $U$ to dominate over velocity dependent corrections, at least at moderately low speeds, so the above model, although simple, may give a good quantitative account of long range vortex interactions. We choose to study type II vortices because these provide a simple, clear-cut dynamic problem: vortex scattering. Type I dynamics is slightly more complicated in that vortices can scatter or form bound states depending on the initial conditions. The coupling chosen for the type II numerical simulations of [9] is $\mu^{2}=2$, a choice which we follow for purposes of comparison. In the Lagrangian (35), the constants $q$ and $m$ are already known for $\mu^{2}=2$, but the vortex mass $M$ is not. Rather than attempt to calculate $M$ from our numerical solution, we use the careful numerical analysis of Rebbi and Jacobs [8]. Unfortunately, they found $M$ for each of a regular sequence of $\mu$ values, rather than $\mu^{2}$ values, so the $\mu=\sqrt{2}$ value is not quoted. However, a graph of $\mu$ against $M$ is very nearly linear, so we use linear interpolation to estimate the $\mu=1.41421 \ldots$ mass from the $\mu=1.4$ and $\mu=1.5$ masses given. The result is $M=1.51230 \pi$. The potential for this coupling is plotted in figure 1 .

From the plot of $U(r)$ we see that all trajectories which do not encroach on the interior region $r<r_{c}$ are scattering trajectories. By time-translation and rotational symmetries, we can, without loss of generality, take the point of closest approach (at which $r=r_{0}$ say) to lie on the $\vartheta=0$ ray and occur at time $t=0$. It is then straightforward to show that $\lim _{t \rightarrow \infty} \vartheta$ is

$$
\vartheta_{\infty}=J^{2} \int_{r_{0}}^{\infty} \frac{d r}{r^{2}}\left[\frac{4}{M}\left(U\left(r_{0}\right)-U(r)\right)+J^{2}\left(\frac{1}{r_{0}^{2}}-\frac{1}{r^{2}}\right)\right]^{-\frac{1}{2}}
$$

where $J=r^{2} \dot{\vartheta}$ is the conserved angular momentum conjugate to $\vartheta$. The deflection angle $\Theta$ is $\pi-2 \vartheta_{\infty}$. Solving the scattering problem then amounts to numerically approximating this integral. Note that there is an integrable singularity in the integrand at $r=r_{0}$. This presents no problem in principle, but it must be treated carefully in any numerical algorithm. Schematically, we handle the integral as follows,

$$
\begin{aligned}
\vartheta_{\infty} & =\int_{r_{0}}^{r_{0}+\delta}+\int_{r_{0}+\delta}^{\Delta}+\int_{\Delta}^{\infty} \\
& \approx \vartheta_{\delta}+\vartheta_{\mathrm{NC}}+\vartheta_{\Delta},
\end{aligned}
$$

where $\delta$ is small $(\delta=0.1)$ and $\Delta$ is large $(\Delta=15)$. The contribution $\vartheta_{\delta}$ is calculated by Taylor expansion of the integrand about $r=r_{0}$, while $\vartheta_{\text {num }}$ is evaluated using the Newton-Cotes rule. At large $r$ the potential falls off exponentially, so for $r>\Delta$ we set $U \equiv 0$ and calculate $\vartheta_{\Delta}$ in the free vortex approximation.

To make comparison with the numerical simulations described in [9] we calculate $\Theta$ as a function of impact parameter $b$ for scattering at impact speeds $v_{\infty}=0.1,0.2,0.3$ and 0.4 . The connexion between $\left(b, v_{\infty}\right)$ and $\left(r_{0}, J\right)$, the parameters used in (36) is found using energy and angular momentum conservation. One might worry that at high $v_{\infty}$ and low $b$ the vortices will penetrate the $r<r_{c}$ zone and become unrealistically captured. In fact even in a head on collision, the speed required for this is greater than 0.4 , so the problem is never encountered. The results are shown in figure 2. As one would expect, the approximation fares reasonably well for large impact parameters and moderate speeds, but less well in scattering processes where the vortices approach one another closely. The fit to the numerical simulations of [9] could be improved by adjusting the values of $q$ and $m$, a procedure which we eschew on the grounds that it would corrupt the deductive nature of the model. 

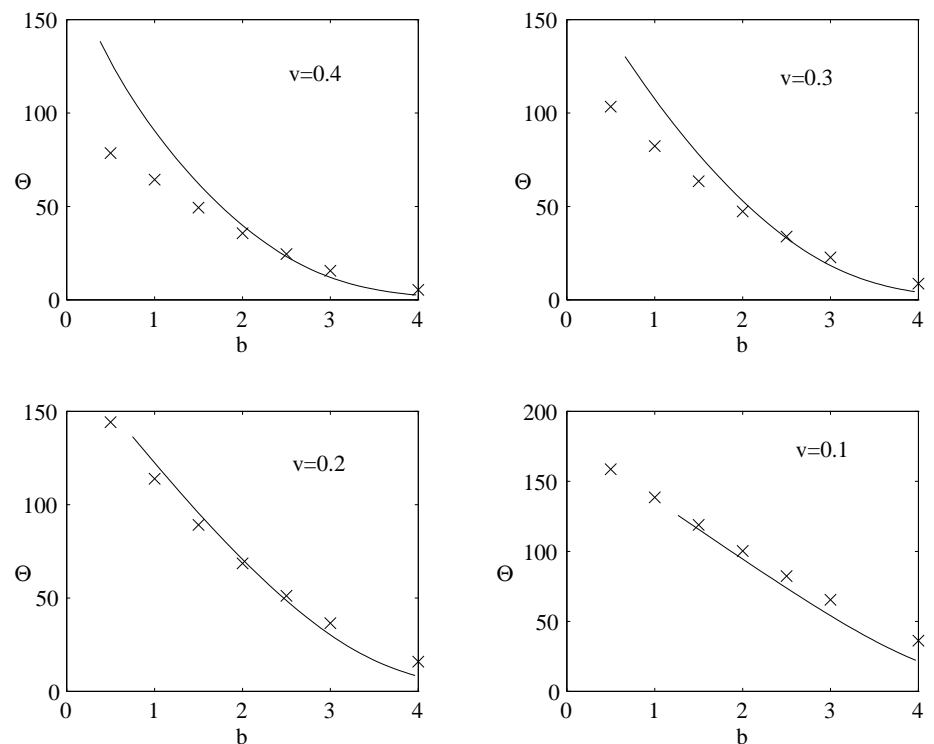

Figure 2: The scattering of $\mu^{2}=2$ (type II) vortices: deflection angle $\Theta$ versus impact parameter $b$ at four different impact speeds. The solid curves were produced using the point source approximation, the crosses by numerical simulation of the full field equations [9].

\section{Conclusion}

In this paper we have presented a point source formalism for long range vortex dynamics. We used this framework to rederive the static intervortex potential from a new perspective and solved the scattering problem for $\mu^{2}=2$ vortices, finding reasonable agreement with numerical simulations, despite the simplicity of the mechanical model. It would be straightforward to apply the method to other situations of interest: to derive the asymptotic forces between a vortex-antivortex pair, or higher winding conglomerations (in the type I regime), or larger collections of vortices for example.

A less straightforward extension of the present work concerns the long range interactions of critically coupled vortices. These exert no forces on one another when at rest, but do affect one another when in relative motion. So the scattering of critically coupled vortices is highly nontrivial, as evidenced by numerical simulations [9] and a partly numerical implementation of the geodesic approximation [7]. In the case of Yang-Mills-Higgs theory, another field theory the scattering of whose critically coupled solitons (BPS monopoles) has been extensively studied using the geodesic approximation, Manton has devised a method for finding long range velocity dependent forces within the point particle approximation [10]. The idea is to calculate the interaction of one point source with the retarded potential generated by another moving along some trajectory. For BPS monopoles, this led to a formula for the asymptotic metric on the two monopole moduli space, which turned out to be in precise agreement with the asymptotic form of the Atiyah-Hitchin metric 11. The method has been adapted to several models [12, 13], but always where the linearized theory is massless. In the vortex case, the linearized theory is massive, so the nontrivial problem is to find a suitable substitute for ordinary retarded potentials (field disturbances no longer travel uniformly at the speed of light, so standard retarded potentials are not appropriate). If velocity dependent intervortex forces can be derived by this means, one could deduce the asymptotic form of the metric on the two vortex moduli space, at present known only numerically. 
Acknowledgments: I would like to thank Bernd Schroers for many valuable conversations. This work developed partly while I was a research student at the University of Durham, England, financially supported by the UK Particle Physics and Astronomy Research Council.

\section{References}

[1] P.W. Higgs, Phys. Rev. 145 (1966) 1156.

[2] A. Vilenkin, Phys. Rep. 121 (1985) 263.

[3] L.M.A. Bettencourt and R.J. Rivers, Phys. Rev. D51 (1995) 1842.

[4] H.B. Nielsen and P. Olesen, Nucl. Phys. B61 (1973) 45.

[5] M. Abramowitz and I.A. Stegun (eds), Pocketbook of Mathematical Functions (Verlag Harri Deutsch, Frankfurt, Germany 1984).

[6] E.B. Bogomol'nyi, Sov. J. Nucl. Phys. 24 (1976) 449.

[7] T.M. Samols, Commun. Math. Phys. 145 (1992) 149.

[8] L. Jacobs and C. Rebbi, Phys. Rev. B19 (1979) 4486.

[9] E. Myers, C. Rebbi and R. Strilka, Phys. Rev. D45 (1992) 1355.

[10] N.S. Manton, Phys. Lett. 154B (1985) 397 and Phys. Lett. 157B (1985) 475 (errata).

[11] M.F. Atiyah and N.J. Hitchin, The Geometry and Dynamics of Magnetic Monopoles (Princeton University Press, Princeton, USA, 1988).

[12] B.J. Schroers, Z. Phys. C61 (1994) 479.

[13] G.W. Gibbons and P.J. Ruback, Phys. Rev. Lett. 57 (1986) 1492. 